\title{
Efficient Series Expansions of the Temperature Field in Dry Surface Grinding for Usual Heat Flux Profiles
}

\author{
Juan Luis González-Santander \\ Universidad Católica de Valencia "San Vicente Mártir", C/Guillem de Castro 96, 46001 Valencia, Spain \\ Correspondence should be addressed to Juan Luis González-Santander; martinez.gonzalez@ucv.es
}

Received 16 May 2017; Revised 1 November 2017; Accepted 9 November 2017; Published 28 December 2017

Academic Editor: Michael Vynnycky

Copyright (C) 2017 Juan Luis González-Santander. This is an open access article distributed under the Creative Commons Attribution License, which permits unrestricted use, distribution, and reproduction in any medium, provided the original work is properly cited.

In the framework of Jaeger's model for heat transfer in dry surface grinding, series expansions for calculating the temperature field, assuming constant, linear, triangular, and parabolic heat flux profiles entering into the workpiece, are derived. The numerical evaluation of these series is considerably faster than the numerical integration of Jaeger's formula and as accurate as the latter. Also, considering a constant heat flux profile, a numerical procedure is proposed for the computation of the maximum temperature as a function of the Peclet number and the depth below the surface. This numerical procedure has been used to evaluate the accuracy of Takazawa's approximation.

\section{Introduction}

Straight grinding is a machining process that produces a smooth finish on a flat surface of a workpiece. In this process, there are hard abrasive grits stuck to the peripheral area of the grinding wheel, which perform the cutting when it rotates at high speed, removing the surface layer of the workpiece (see Figure 1). Also, the workpiece moves at a certain feed rate $v_{d}$ with respect to the wheel and contacts the latter at the grinding area, which is $2 \ell$ wide (see Table 1 for the nomenclature used). Jaeger's model [1, Sect. 10.7.VII] is commonly used to calculate the temperature field in dry grinding. In this model, a two-dimensional approach is considered, in which the coordinate system is fixed to the wheel and centered on the middle of the grinding area, as shown in Figure 1. In surface grinding, the cutting depth $a$ is small, whereby the grinding area is assumed to be flat in Jaeger's model.

The time-dependent temperature field $T(t, x, y)$ of the workpiece in Jaeger's model satisfies the convective heat equation $[1, \$ 1.7(2)]$

$$
\frac{\partial T}{\partial t}=k\left(\frac{\partial^{2} T}{\partial x^{2}}+\frac{\partial^{2} T}{\partial y^{2}}\right)+v_{d} \frac{\partial T}{\partial x}
$$

subjected to the initial condition,

$$
T(0, x, y)=T_{\infty},
$$

and to the boundary condition

$$
k_{0} \frac{\partial T}{\partial y}(t, x, 0)=-q f(x) \theta(\ell-x) \theta(\ell+x),
$$

where $\theta(x)$ denotes the Heaviside function and $f(x)$ is the dimensionless heat flux profile going to the workpiece, which is normalized to unity,

$$
\frac{1}{2 \ell} \int_{-\ell}^{\ell} f(x) d x=1 .
$$

The temperature field in the stationary regime is reached when $\partial T / \partial t=0$; thus it does not depend on $t$, and it is denoted as $T(x, y)$. In the stationary regime, the solution of the above equations (1)-(3) is given by [2, Eqn. 6-1]

$$
\begin{gathered}
T(x, y)=T_{\infty}+\frac{q}{\pi k_{0}} \int_{-\ell}^{\ell} f(u) \exp \left(\frac{-v_{d}(x-u)}{2 k}\right) \\
\cdot K_{0}\left(\frac{v_{d}}{2 k} \sqrt{(x-u)^{2}+y^{2}}\right) d u,
\end{gathered}
$$

where $K_{0}(z)$ denotes the modified Bessel function of the second kind of zero order [3, Chap. 51].

Figure 2 shows the usual heat flux profiles $f(x)$ considered in the literature: constant $[4,5]$, linear [6-8], triangular $[9,10]$, and parabolic $[11,12]$. 
TABLE 1: Nomenclature of symbols.

\begin{tabular}{lcc}
\hline Symbol & Meaning & \multicolumn{1}{c}{ SI units } \\
\hline$k_{0}$ & Thermal conductivity & $\mathrm{W} \mathrm{m}^{-1} \mathrm{~K}^{-1}$ \\
$k$ & Thermal diffusivity & $\mathrm{m}^{2} \mathrm{~s}^{-1}$ \\
$T$ & Workpiece temperature & $\mathrm{K}$ \\
$T_{\infty}$ & Room temperature & $\mathrm{K}$ \\
$v_{d}$ & Feed rate & $\mathrm{m} \mathrm{s}^{-1}$ \\
$2 \ell$ & Contact length & $\mathrm{m}$ \\
$q$ & Average heat flux within contact length & $\mathrm{W} \mathrm{m}^{-2}$ \\
$a$ & Cutting depth & $\mathrm{m}$ \\
\hline
\end{tabular}

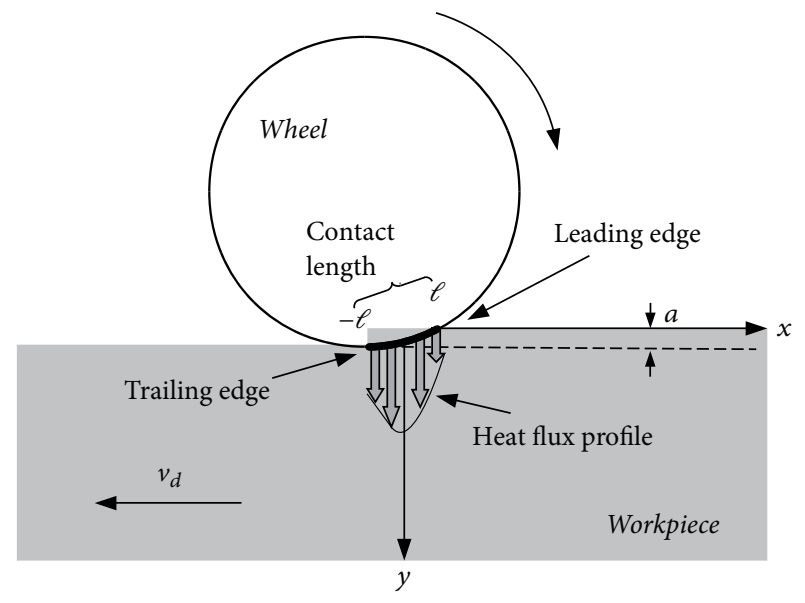

FIGURE 1: Straight grinding setup.

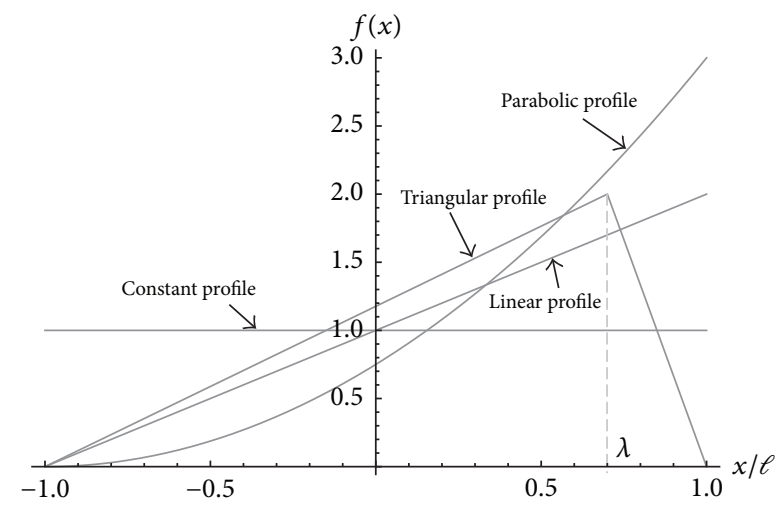

FIGURE 2: Usual heat flux profiles considered in the literature.

We can rewrite (5) in dimensionless form, setting the following dimensionless quantities: $\mathscr{T}=\pi k_{0}\left(T-T_{\infty}\right) /(q s)$, $X=x / s, Y=y / s$, and $L=\ell / s$ (Peclet number), where

$$
s=\frac{2 k}{v_{d}}
$$

is a characteristic length. Thereby,

$$
\mathscr{T}(X, Y)=\int_{X-L}^{X+L} f(s[X-u]) e^{-u} K_{0}\left(\sqrt{u^{2}+Y^{2}}\right) d u
$$

On the one hand, on the surface, that is, $Y=0$, (7) is reduced to

$$
\mathscr{T}(X, 0)=\int_{X-L}^{X+L} f(s[X-u]) e^{-u} K_{0}(|u|) d u,
$$

and when $f(x)$ is an analytic function, closed form expressions for the dimensionless temperature on the surface can be obtained [13]:

$$
\mathscr{T}(X, 0)=\left.\sum_{n=0}^{\infty} \frac{f^{(n)}(s X)}{n !}(-s)^{n} \operatorname{Jg}_{n}(u)\right|_{u=X-L} ^{X+L},
$$

where

$$
\begin{aligned}
& \operatorname{Jg}_{n}(x) \\
& = \begin{cases}e^{x} x^{n+1}\left\{K_{0}(|x|) \Psi_{n}(x)+K_{1}(|x|) \Phi_{n}(x)\right\}, & x \neq 0 \\
0, & x=0\end{cases}
\end{aligned}
$$

where

$$
\begin{aligned}
& \Psi_{n}(x)=\frac{1}{n+1}\left[\begin{array}{c}
1 \\
\frac{1}{2}-n
\end{array}\right. \\
& \quad-\frac{n}{2 n+1}{ }_{3} F_{1}\left(\begin{array}{c}
1,1-n,-1-n \\
2 x
\end{array}\right), \\
& \Phi_{n}(x)=\frac{\operatorname{sgn}(x)}{2 n+1}{ }_{3} F_{1}\left(\begin{array}{c|c}
1,-n,-n & 1 \\
-n+\frac{1}{2} & \frac{1}{2 x}
\end{array}\right),
\end{aligned}
$$

polynomials in $1 / x$. For instance, for a constant heat flux profile,

$$
\mathscr{T}_{0}(X, 0)=\left.\operatorname{Jg}_{0}(u)\right|_{u=-X-L} ^{-X+L}
$$

and according to (10)-(11), we have

$$
\begin{aligned}
& \operatorname{Jg}_{0}(x) \\
& \quad= \begin{cases}x e^{x}\left[K_{0}(|x|)+\operatorname{sign}(x) K_{1}(|x|)\right], & x \neq 0 \\
0, & x=0 .\end{cases}
\end{aligned}
$$

It is worth noting that the $x$-axis given in [13] is just in the opposite direction as the one given in Figure 1. For coherence, 
all the formulas in this paper are referred to the coordinate system given in Figure 1. From the results given in [13], similar formulas to (12) can be derived for other heat flux profiles normalized to unity (4). For instance, considering a linear heat flux profile, we have

$$
\mathscr{T}_{1}(X, 0)=\frac{1}{2 L}\left\{(X+L) \operatorname{Jg}_{0}(u)+\operatorname{Jg}_{1}(u)\right\}_{u=-X-L}^{-X+L} .
$$

Also, for a parabolic heat flux profile, we obtain

$$
\begin{aligned}
& \mathscr{T}_{2}(X, 0)=\frac{3}{4 L^{2}}\left[(X+L)^{2} \operatorname{Jg}_{0}(u)-2(X+L) \operatorname{Jg}_{1}(u)\right. \\
& \left.\quad+\operatorname{Jg}_{2}(u)\right]_{u=-X-L}^{-X+L}
\end{aligned}
$$

and, for a triangular one,

$$
\begin{aligned}
\mathscr{T}_{\Delta} & (X, 0) \\
& =2\left(\frac{H_{L}(X)}{1-\lambda}-\frac{H_{-L}(X)}{1+\lambda}+\frac{2 \lambda}{\lambda^{2}-1} H_{\lambda L}(X)\right),
\end{aligned}
$$

where

$$
H_{\Lambda}(X)=\operatorname{Jg}_{0}(\Lambda-X)\left(1-\frac{X}{\Lambda}\right)-\frac{1}{\Lambda} \operatorname{Jg}_{1}(\Lambda-X) .
$$

On the other hand, considering a constant heat flux profile, (18) is reduced to [14]

$$
\mathscr{T}_{0}(X, Y)=\int_{X-L}^{X+L} e^{-u} K_{0}\left(\sqrt{u^{2}+Y^{2}}\right) d u .
$$

Takazawa [15] provides the following approximation to (18) for the maximum temperature reached at a given depth $Y$ below the surface,

$$
\mathscr{T}_{0, \text { max }}(Y, L) \approx 3.1 L^{0.53} \exp \left(-0.69 L^{-0.37} Y\right) .
$$

Expression (19) comes from the numerical evaluation of (18) and a parameter fitting of the maximum temperature. Takazawa uses (19) to estimate how the hardness of the workpiece changes beneath its surface

The scope of this paper is two-folded. On the one hand, we derive some series expansions to calculate the integral given in (7) for the heat flux profiles considered above (see Figure 2). It turns out that the numerical evaluation of $\mathscr{T}(X, Y)$ by using these series is considerably faster than the numerical integration of (7). On the other hand, we will provide a numerical procedure to calculate $\mathscr{T}_{0 \text {,max }}(Y, L)$ that allow us to evaluate the accuracy of Takazawa's approximation (19).

This paper is organized as follows. Section 2 provides particular expressions of (7) for the different heat flux profiles considered above, that is, constant, linear, triangular, and parabolic. Section 3 is devoted to the calculation of the Taylor series of $K_{v}\left(\sqrt{u^{2}+y^{2}}\right)\left(u^{2}+y^{2}\right)^{-v / 2}$, whereby, taking $v=0$, we get the main factor of the integrand given in (7). In Section 4 , we use the result of the previous section to express $\mathscr{T}(X, Y)$ as a series expansion for the different heat flux profiles considered in this paper. From these series expansions, we can recover the results given in (12) and (14)-(16) for the dimensionless temperature on the surface, that is, $\mathscr{T}(X, 0)$. As an example of this consistency test, we derive the latter for the case of a constant heat flux profile. Section 5 describes a quite efficient numerical procedure to calculate $\mathscr{T}_{0 \text {, max }}(Y, L)$. In Section 6, we present some numerical simulations in order to compare the performance of the numerical integration of Jaeger's formula (7) with the series expansions derived in Section 4 for the different heat flux profiles considered. Also, we evaluate the accuracy of Takazawa's approximation (19) as a function of $Y$ and $L$. Finally, we collect our conclusions in Section 7.

\section{Temperature Field for Usual Heat Flux Profiles}

Consider now that $f(x)$ is an analytic function within the contact area between the workpiece and the wheel; that is, $x \in[-\ell, \ell]$,

$$
f(s X-s u)=\sum_{m=0}^{\infty} \frac{f^{(m)}(s X)}{m !}(-s u)^{m} .
$$

Therefore, inserting (20) in (7)

$$
\mathscr{T}(X, Y)=\left.\sum_{m=0}^{\infty} \frac{f^{(m)}(s X)}{m !}(-s)^{m} J_{m}(u)\right|_{u=X-L} ^{X+L},
$$

where we have set

$$
\begin{array}{r}
J_{m}(x)=\int_{0}^{x} u^{m} e^{-u} K_{0}\left(\sqrt{u^{2}+Y^{2}}\right) d u, \\
\quad m=0,1,2, \ldots
\end{array}
$$

For a constant heat flux profile,

$$
f_{0}(x)=1
$$

thus (21) is reduced to

$$
\mathscr{T}_{0}(X, Y)=\left.J_{0}(u)\right|_{u=X-L} ^{X+L}
$$

Taking into account the normalization condition (4), for a linear heat flux profile, we have

$$
f_{1}(x)=1+\frac{x}{\ell}
$$

hence

$$
\mathscr{T}_{1}(X, Y)=\frac{1}{L}\left[(X+L) J_{0}(u)-J_{1}(u)\right]_{u=X-L}^{X+L} .
$$

For a triangular heat profile

$$
f_{\Delta}(x)= \begin{cases}\frac{2}{1+\lambda}\left(1+\frac{x}{\ell}\right), & x \in\left(-\ell, \ell_{\max }\right) \\ \frac{2}{1-\lambda}\left(1-\frac{x}{\ell}\right), & x \in\left(\ell_{\max }, \ell\right),\end{cases}
$$


where $\ell_{\max } \in[-\ell, \ell]$ denotes the location of the apex in the triangular profile and $\lambda$ is the following dimensionless parameter (see Figure 2):

$$
\lambda=\frac{\ell_{\max }}{\ell} \in[-1,1] .
$$

Notice that when the heat flux occurs in an arbitrary interval, say $x \in(a, b)$, the dimensionless temperature is given by

$$
\begin{aligned}
\mathscr{T} & (X, Y) \\
& =\int_{X-B}^{X-A} f(s[X-u]) e^{-u} K_{0}\left(\sqrt{u^{2}+Y^{2}}\right) d u,
\end{aligned}
$$

where $A=a / s$ and $B=b / s$ are both dimensionless parameters. Since the heat equation is linear, the temperature field that $f_{\Delta}(x)$ generates in the workpiece is given by the superposition of both parts of (27). Therefore, taking into account (29), we have

$$
\begin{aligned}
& \mathscr{T}_{\Delta}(X, Y)=\frac{2}{1+\lambda} \\
& \cdot \int_{X-\lambda L}^{X+L}\left(1+\frac{X-u}{L}\right) e^{-u} K_{0}\left(\sqrt{u^{2}+Y^{2}}\right) d u \\
& +\frac{2}{1-\lambda} \\
& \cdot \int_{X-L}^{X-\lambda L}\left(1-\frac{X-u}{L}\right) e^{-u} K_{0}\left(\sqrt{u^{2}+Y^{2}}\right) d u .
\end{aligned}
$$

We can rewrite $\mathscr{T}_{\Delta}(X, Y)$ as follows:

$$
\mathscr{T}_{\Delta}(X, Y)=\mathscr{T}_{\lambda, L}(X, Y)-\mathscr{T}_{-\lambda,-L}(X, Y),
$$

where we have set

$$
\begin{aligned}
& \mathscr{T}_{\lambda, L}(X, Y) \\
& \quad=\frac{2}{L(1+\lambda)}\left\{(X+L) J_{0}(u)-J_{1}(u)\right\}_{u=X-\lambda L}^{X+L} .
\end{aligned}
$$

Finally, for a parabolic heat flux profile,

$$
f_{2}(x)=\frac{3}{4}\left(1+\frac{x}{\ell}\right)^{2}
$$

whereby

$$
\begin{aligned}
& \mathscr{T}_{2}(X, Y)=\frac{3}{4 L^{2}}\left[(X+L)^{2} J_{0}(u)-2(X+L) J_{1}(u)\right. \\
& \left.\quad+J_{2}(u)\right]_{u=X-L}^{X+L} .
\end{aligned}
$$

\section{Taylor Expansion of the Integrand}

In order to calculate $J_{m}(x)$, which is defined as an integral in (22), consider this more general form of the same integral,

$$
\left.\mathcal{I}_{m}^{ \pm}(\nu, y, u)\right|_{u=a} ^{b}=\int_{a}^{b} e^{ \pm u} u^{m} \frac{K_{\nu}\left(\sqrt{u^{2}+y^{2}}\right)}{\left(u^{2}+y^{2}\right)^{v / 2}} d u ;
$$

thereby

$$
\left.J_{m}(u)\right|_{u=a} ^{b}=\left.\mathscr{I}_{m}^{-}(0, Y, u)\right|_{u=a} ^{b} .
$$

To expand $\mathscr{I}_{m}^{ \pm}(\nu, z, a, b)$ in series, we calculate first the Taylor series of $K_{\nu}\left(\sqrt{u^{2}+y^{2}}\right)\left(u^{2}+y^{2}\right)^{-v / 2}$. For this purpose, according to [16, Eqn. 1.14.1(4)], we have this formula for the $n$th derivative

$$
D^{n}\left[z^{-v / 2} K_{\nu}(\sqrt{z})\right]=\left(-\frac{1}{2}\right)^{n}\left(\frac{1}{\sqrt{z}}\right)^{n+\nu} K_{n+\nu}(\sqrt{z}) .
$$

Since the Taylor expansion of an analytic function is

$$
f(x+h)=\sum_{n=0}^{\infty} \frac{f^{(n)}(h)}{n !} x^{n}=\sum_{n=0}^{\infty} \frac{f^{(n)}(x)}{n !} h^{n},
$$

according to (37), we have

$$
\begin{aligned}
\frac{K_{\nu}(\sqrt{x+h})}{(x+h)^{v / 2}} & =\frac{1}{h^{v / 2}} \sum_{n=0}^{\infty} \frac{1}{n !}\left(\frac{-1}{2 \sqrt{h}}\right)^{n} K_{n+\nu}(\sqrt{h}) x^{n} \\
& =\frac{1}{x^{v / 2}} \sum_{n=0}^{\infty} \frac{1}{n !}\left(\frac{-1}{2 \sqrt{x}}\right)^{n} K_{n+\nu}(\sqrt{x}) h^{n} .
\end{aligned}
$$

To calculate the radius of convergence of (39), let us set

$$
b_{n}=\frac{1}{n !}\left(\frac{-1}{2 \sqrt{h}}\right)^{n} K_{n+\nu}(\sqrt{h}) x^{n}
$$

so, applying the ratio test, we have to determinate when $R<1$, where

$$
R=\lim _{n \rightarrow \infty}\left|\frac{b_{n+1}}{b_{n}}\right|=\frac{x}{2 \sqrt{h}^{n \rightarrow \infty}} \lim _{n \rightarrow 1} \frac{1}{n+1} \frac{K_{n+\nu+1}(\sqrt{x})}{K_{n+\nu}(\sqrt{x})} .
$$

By using the asymptotic formula [17, Eqn. 10.41.2],

$$
K_{\mu}(z) \approx \sqrt{\frac{\pi}{2 \mu}}\left(\frac{e z}{2 \mu}\right)^{-\mu}, \quad \mu \longrightarrow \infty,
$$

we calculate (42) as

$$
\begin{aligned}
R & =\frac{x}{h} e^{-1} \lim _{n \rightarrow \infty} \frac{\sqrt{n+v+1} \sqrt{n+\nu}}{n+1}\left(1+\frac{1}{n+\nu}\right)^{n+\nu} \\
& =\frac{x}{h}
\end{aligned}
$$

where we have taken the definition of number $e=\lim _{n \rightarrow \infty}(1+$ $1 / n)^{n}$ [18, Eqn. 1.2]. Therefore, (39) converges absolutely when $x<h$. Notice that we can convert (39) into (40) exchanging $x$ and $h$, so (40) converges absolutely when $x>h$. 
Finally, taking $h=u^{2}$ and $x=y^{2}$ in (39) and (40), bearing in mind what we have said about the radius of convergence, we arrive at

$$
\begin{aligned}
& \frac{K_{\nu}\left(\sqrt{u^{2}+y^{2}}\right)}{\left(u^{2}+y^{2}\right)^{v / 2}} \\
& = \begin{cases}\frac{1}{|u|^{\nu}} \sum_{n=0}^{\infty} \frac{1}{n !}\left(\frac{-y^{2}}{2|u|}\right)^{n} K_{n+v}(|u|), & |y|<|u| \\
\frac{1}{|y|^{\nu}} \sum_{n=0}^{\infty} \frac{1}{n !}\left(\frac{-u^{2}}{2|y|}\right)^{n} K_{n+v}(|y|), & |y|>|u| .\end{cases}
\end{aligned}
$$

The case $|y|=|u|$ is not essential for our purpose, since we are going to insert in (35) the expansions given in (45) and then integrate term by term.

\section{Series Expansion of the Temperature Field}

In order to insert (45) in (35) and integrate term by term, let us define the following functions:

$$
\begin{aligned}
& \left.\mathscr{I}_{m, \text { upper }}^{ \pm}(\nu, y, u)\right|_{u=a} ^{b} \\
& \quad=\sum_{n=0}^{\infty} \frac{\left(-y^{2} / 2\right)^{n}}{n !} \int_{a}^{b} e^{ \pm u} u^{m} \frac{K_{n+\nu}(|u|)}{|u|^{n+\nu}} d u, \quad|u|>y, \\
& \left.\mathscr{I}_{m, \text { lower }}^{ \pm}(\nu, y, u)\right|_{u=a} ^{b} \\
& =\frac{1}{y^{\nu}} \sum_{n=0}^{\infty} \frac{(-2 y)^{-n}}{n !} K_{n+\nu}(y) \int_{a}^{b} e^{ \pm u} u^{2 n+m} d u,
\end{aligned}
$$

$$
|u|<y .
$$

We consider hereafter $a<b$. Also, according to Figure 1 , $y \geq 0$; thus, we have dropped the absolute value for $y$ in (46) and (47).

4.1. Calculation of $\mathscr{I}_{m \text {,upper. }}^{ \pm}$For the calculation of the integral given in (46), define the following function:

$$
\left.h_{m}^{ \pm}(\alpha, u)\right|_{u=a} ^{b}=\int_{a}^{b} e^{ \pm u} u^{m} \frac{K_{\alpha}(|u|)}{|u|^{\alpha}} d u,
$$

so that, we can rewrite (46) as

$$
\begin{aligned}
& \left.\mathscr{J}_{m, \text { upper }}^{ \pm}(v, y, u)\right|_{u=a} ^{b} \\
& \quad=\left.\sum_{n=0}^{\infty} \frac{\left(-y^{2} / 2\right)^{n}}{n !} h_{m}^{ \pm}(n+v, u)\right|_{u=a} ^{b} .
\end{aligned}
$$

First, note that, performing the change of variables $u \rightarrow$ $-u$ on the LHS of (48), we obtain

$$
\begin{aligned}
\left.h_{m}^{ \pm}(\alpha, u)\right|_{u=a} ^{b} & =(-1)^{m+1} \int_{-a}^{-b} e^{\mp u} u^{m} \frac{K_{\alpha}(|u|)}{|u|^{\alpha}} d u \\
& =\left.(-1)^{m+1} h_{m}^{\mp}(\alpha, u)\right|_{u=-a} ^{-b} ;
\end{aligned}
$$

thus

$$
\begin{aligned}
& \left.\mathscr{I}_{m, \text { upper }}^{ \pm}(\nu, y, u)\right|_{u=a} ^{b} \\
& \quad=\left.(-1)^{m+1} \mathscr{I}_{m, \text { upper }}^{\mp}(\nu, y, u)\right|_{u=-a} ^{-b} .
\end{aligned}
$$

Also, when $a, b>0$,

$$
\begin{aligned}
\left.h_{m}^{ \pm}(\alpha, u)\right|_{u=a} ^{b} & =\int_{a}^{b} e^{ \pm u} u^{m-\alpha} K_{\alpha}(u) d u \\
& =\int_{a}^{b} e^{ \pm u} u^{m+\beta} K_{\beta}(u) d u
\end{aligned}
$$

where we have set $\beta=-\alpha$ and applied the property $K_{\nu}(z)=$ $K_{-v}(z)$ [19, Eqn. 5.7.10]. For the calculation of $h_{m}^{ \pm}(\alpha, u)$, we found in the literature [13]

$$
\begin{aligned}
& \int_{0}^{z} e^{ \pm x} x^{\nu+n} K_{\nu}(x) d x=e^{ \pm z} z^{\nu+n+1} \times\left\{\frac{K_{v}(z)}{2 \nu+n+1}[1\right. \\
& -\frac{n}{2 v+2 n+1} \\
& \left.\cdot{ }_{3} F_{1}\left(\begin{array}{c|c}
1,1-n,-1-n-2 v & \pm 1 \\
\frac{1}{2}-n-v & \frac{ \pm 1}{2 z}
\end{array}\right)\right] \\
& \left. \pm \frac{K_{1+v}(z)}{2 v+2 n+1}{ }_{3} F_{1}\left(\begin{array}{c|c}
1,-n,-n-2 v & \pm 1 \\
-n+\frac{1}{2}-v & \frac{ \pm 1}{2 z}
\end{array}\right)\right\} \\
& +(\mp 1)^{n+1} 2^{\nu+n} \frac{n ! \Gamma(\nu+n+1) \Gamma(2 v+n+1)}{\Gamma(2 v+2 n+2)} \text {. }
\end{aligned}
$$

Therefore,

$$
\begin{gathered}
\left.h_{m}^{ \pm}(\alpha, u)\right|_{u=a} ^{b}=e^{ \pm u} u^{1+m-\alpha}\left\{\frac{K_{\alpha}(u)}{1+m-2 \alpha}[1\right. \\
\left.\left.-\frac{m P_{m}^{\alpha}(u)}{1+2(m-\alpha)}\right] \pm \frac{K_{\alpha-1}(u) Q_{m}^{\alpha}(u)}{1+2(m-\alpha)}\right\}\left.\right|_{u=a} ^{b},
\end{gathered}
$$

$$
a, b>0,
$$

where we have defined the following polynomials in $1 / u$ :

$$
\begin{aligned}
P_{m}^{\alpha}(u) & ={ }_{3} F_{1}\left(\begin{array}{c|c}
1,1-m, 2 \alpha-m-1 \\
\frac{1}{2}-m+\alpha
\end{array} \mid \frac{ \pm 1}{2 u}\right) \\
& =\sum_{s=0}^{\infty} \frac{(1-m)_{s}(2 \alpha-m-1)_{s}}{(1 / 2-m+\alpha)_{s}}\left(\frac{ \pm 1}{2 u}\right)^{s}, \\
Q_{m}^{\alpha}(u) & ={ }_{3} F_{1}\left(\begin{array}{c}
1,-m, 2 \alpha-m \\
\frac{1}{2}-m+\alpha
\end{array} \mid \frac{ \pm 1}{2 u}\right) \\
& =\sum_{s=0}^{\infty} \frac{(-m)_{s}(2 \alpha-m)_{s}}{(1 / 2-m+\alpha)_{s}}\left(\frac{ \pm 1}{2 u}\right)^{s} .
\end{aligned}
$$

Note that the degree of polynomial $P_{m}^{\alpha}$ is $m+1$ and of polynomial $Q_{m}^{\alpha}$ is $m$. According to Section 2, we need to calculate $J_{m}$ for $m=0,1,2$; thus we are going to derive 
particular expressions of $\mathscr{I}_{m \text {, upper }}^{ \pm}$for $v=0$ and $m=0,1,2$, when $a, b>0$. Thereby, taking $\nu=0,(49)$ is reduced to

$$
\left.\mathscr{J}_{m, \text { upper }}^{ \pm}(0, y, u)\right|_{u=a} ^{b}=\left.\sum_{n=0}^{\infty} \frac{\left(-y^{2} / 2\right)^{n}}{n !} h_{m}^{ \pm}(n, u)\right|_{u=a} ^{b}
$$

In the case $n=0$, according to (54)-(55), we have

$$
\begin{aligned}
&\left.h_{0}^{ \pm}(n, u)\right|_{u=a} ^{b}=\left.e^{ \pm u} u^{1-n} \frac{K_{n}(u) \pm K_{n-1}(u)}{1-2 n}\right|_{u=a} ^{b}, \\
& a, b>0 .
\end{aligned}
$$

Similarly, for $m=1,(54)$ is reduced to

$$
\begin{aligned}
& \left.h_{1}^{ \pm}(n, u)\right|_{u=a} ^{b} \\
& \quad=\left.e^{ \pm u} u^{1-n} \frac{K_{n}(u) u-K_{n-1}(u)(1 \mp u)}{3-2 n}\right|_{u=a} ^{b},
\end{aligned}
$$

Finally, for $m=2$, we arrive at

$$
\left.h_{2}^{ \pm}(n, u)\right|_{u=a} ^{b}=e^{ \pm u} u^{1-n} \times\left.\frac{K_{n}(u)[(3-2 n) u \pm 2] u \pm K_{n-1}(u)\left[(3-2 n) u^{2}+4(1-n)(1 \mp u)\right]}{(3-2 n)(5-2 n)}\right|_{u=a} ^{b}, \quad a, b>0
$$

4.2. Calculation of $\mathscr{J}_{m}^{ \pm}$lower. For the calculation of the integral given in (47), we apply the definition of the lower incomplete gamma function [3, Eqn. 45:3:1]:

$$
\gamma(\nu, x)=\int_{0}^{x} t^{\nu-1} e^{-t} d t
$$

so that, straightforwardly, we obtain

$$
\int_{a}^{b} e^{-u} u^{2 n+m} d u=\left.\gamma(2 n+m+1, u)\right|_{u=a} ^{b}
$$

and, performing the change of variables $u \rightarrow-u$, we have

$$
\int_{a}^{b} e^{u} u^{2 n+m} d u=\left.(-1)^{m+1} \gamma(2 n+m+1, u)\right|_{u=-a} ^{-b}
$$

Taking into account (61) and (62), we rewrite (47) as

$$
\begin{aligned}
& \left.\mathscr{I}_{m, \text { lower }}^{ \pm}(\nu, y, u)\right|_{u=a} ^{b}=\frac{(\mp 1)^{m+1}}{y^{\nu}} \sum_{n=0}^{\infty} \frac{(-2 y)^{-n}}{n !} K_{n+v}(y) \\
& \left.\cdot \gamma(2 n+m+1, u)\right|_{u=\mp a} ^{\mp b},
\end{aligned}
$$

which, for $v=0$, is reduced to

$$
\begin{gathered}
\left.\mathscr{J}_{m, \text { lower }}^{ \pm}(0, y, u)\right|_{u=a} ^{b}=(\mp 1)^{m+1} \sum_{n=0}^{\infty} \frac{(-2 y)^{-n}}{n !} K_{n}(y) \\
\left.\cdot \gamma(2 n+m+1, u)\right|_{u=\mp a} ^{\mp b}, \quad \max (|a|,|b|)<y .
\end{gathered}
$$

4.3. Calculation of $\mathscr{I}_{\mathrm{m}}^{ \pm}$. Applying the property given in (51), we have six different cases for the calculation of $\mathscr{F}_{m}^{ \pm}$in terms of $\mathscr{I}_{m \text {,upper }}^{ \pm}$and $\mathscr{J}_{m \text {,lower }}^{ \pm}$. For the sake of clarity, here we use the following simplified notation: $\left.\mathscr{I}_{m}^{ \pm}\right|_{a} ^{b}=\left.\mathscr{I}_{m}^{ \pm}(\nu, y, u)\right|_{u=a} ^{b}$.

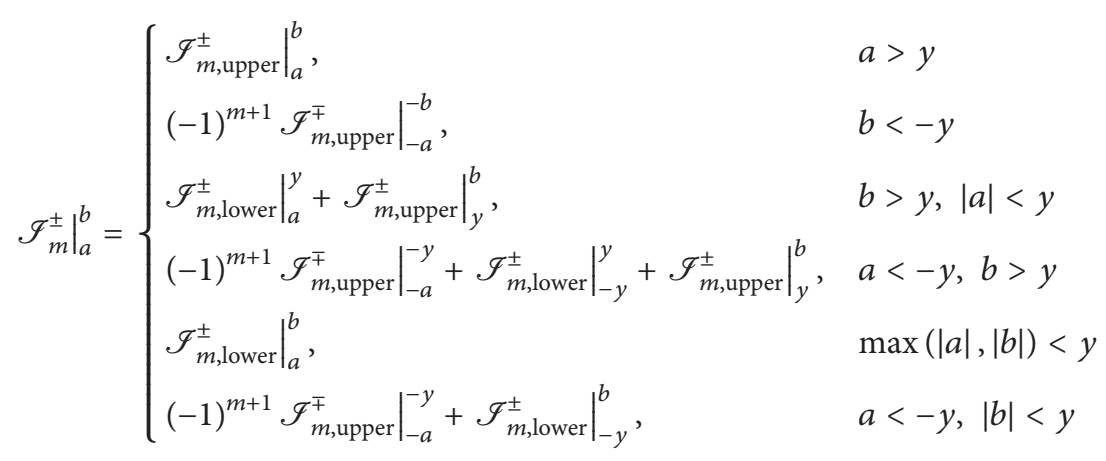

that can be reduced to only three cases, 


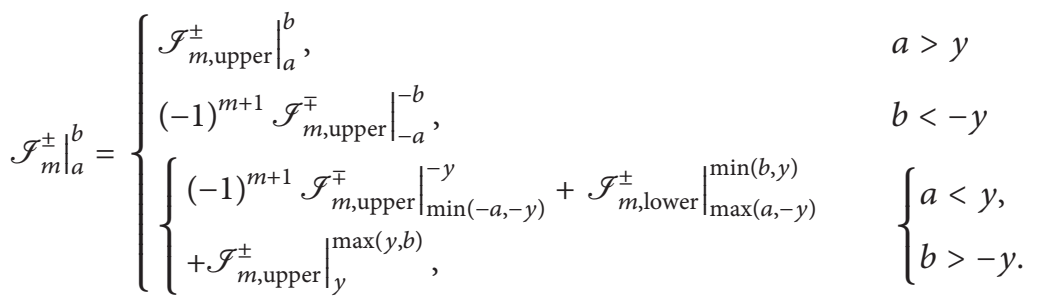

Recalling that $y \geq 0$, note that in (65) the integration limits of $\mathscr{I}_{m \text {,upper }}$ are always both positive, so that we can use (56) in combination with (57), (58), or (59), for its calculation.

4.4. Calculation of $\mathscr{T}(X, Y)$. Now, we collect the previous results in order to calculate the dimensionless temperature field $\mathscr{T}(X, Y)$ for the different heat flux profiles considered in the Introduction. First, consider a constant heat flux profile; thereby, according to (24) and (36), we have

$$
\mathscr{T}_{0}(X, Y)=\left.\mathscr{I}_{0}^{-}(0, Y, u)\right|_{u=X-L} ^{X+L} .
$$

Notice that, taking $m=0$ in (51), we have

$$
\left.\mathscr{I}_{0, \text { upper }}^{ \pm}(\nu, y, u)\right|_{u=a} ^{b}=-\left.\mathscr{I}_{0, \text { upper }}^{\mp}(\nu, y, u)\right|_{u=-a} ^{-b} .
$$

Also, from (56) and (57), we obtain the following series for positive integration limits $a, b$ :

$$
\begin{aligned}
& \left.\mathscr{I}_{0, \text { upper }}^{ \pm}(0, y, u)\right|_{u=a} ^{b} \\
& =\left.\sum_{n=0}^{\infty} \frac{\left(-y^{2} / 2\right)^{n}}{n !} e^{ \pm u} u^{1-n} \frac{K_{n}(u) \pm K_{n-1}(u)}{1-2 n}\right|_{u=a} ^{b}, \\
& a, b>0 .
\end{aligned}
$$

According to (68), we can extend (69) for negative values of the integration limits $a, b$, defining the following function:

$$
\begin{aligned}
& \left.\mathbf{I}_{\text {upper }}^{0}(Y, u)\right|_{u=a} ^{b}=\left.\mathscr{I}_{0, \text { upper }}^{-}(0, Y, u)\right|_{u=a} ^{b}=\operatorname{sgn}(a) \\
& \cdot \sum_{n=0}^{\infty} \frac{\left(-Y^{2} / 2\right)^{n}}{n !} \\
& \left.\cdot e^{-\operatorname{sgn}(a) u} u^{1-n} \frac{K_{n}(u)-\operatorname{sgn}(a) K_{n-1}(u)}{1-2 n}\right|_{u=|a|} ^{|b|}, \\
& a b>0 .
\end{aligned}
$$

Similarly, from (64), define

$$
\begin{aligned}
\left.\mathbf{I}_{\text {lower }}^{0}(Y, u)\right|_{u=a} ^{b} & =\left.\mathscr{I}_{0, \text { lower }}^{-}(0, Y, u)\right|_{u=a} ^{b} \\
& =\left.\sum_{n=0}^{\infty} \frac{K_{n}(Y)}{(-2 Y)^{n} n !} \gamma(2 n+1, u)\right|_{u=a} ^{b} .
\end{aligned}
$$

For a linear heat flux profile, according to (26) and (36), we have

$$
\begin{aligned}
& \mathscr{T}_{1}(X, Y) \\
& \quad=\frac{1}{L}\left[(X+L) \mathscr{I}_{0}^{-}(0, Y, u)-\mathscr{I}_{1}^{-}(0, Y, u)\right]_{u=X-L}^{X+L},
\end{aligned}
$$

and, applying (51), we have

$$
\begin{aligned}
\frac{1}{L}[ & \left.(X+L) \mathscr{I}_{0, \text { upper }}^{ \pm}(0, Y, u)-\mathscr{I}_{1, \text { upper }}^{ \pm}(0, Y, u)\right]_{u=a}^{b} \\
= & \frac{1}{L}\left[-(X+L) \mathscr{F}_{0, \text { upper }}^{\mp}(0, Y, u)\right. \\
& \left.-\mathscr{I}_{1, \text { upper }}^{\mp}(0, Y, u)\right]_{u=a}^{b} .
\end{aligned}
$$

Now, taking into account (56), (57) and (58), we can rewrite (73) as

$$
\begin{aligned}
& \frac{1}{L}\left[(X+L) \mathscr{J}_{0, \text { upper }}^{ \pm}(0, Y, u)-\mathscr{I}_{1, \text { upper }}^{ \pm}(0, Y, u)\right]_{u=a}^{b} \\
& \quad=\frac{1}{L} \sum_{n=0}^{\infty} \frac{\left(-y^{2} / 2\right)^{n}}{n !} e^{ \pm u} u^{1-n} \\
& \quad \times\left[K_{n}(u)\left(\frac{X+L}{1-2 n}-\frac{u}{3-2 n}\right)\right. \\
& \left.\quad+K_{n-1}(u)\left(\frac{1 \mp u}{3-2 n} \pm \frac{X+L}{1-2 n}\right)\right]_{u=a}^{b}, \quad a, b>0 .
\end{aligned}
$$

Again, according to (73), we can extend (74) for negative values of the integration limits $a, b$, defining

$$
\begin{aligned}
& \left.\mathbf{I}_{\text {upper }}^{1}(X, Y, u)\right|_{u=a} ^{b}=\frac{1}{L}\left[(X+L) \mathscr{I}_{0, \text { upper }}^{-}(0, Y, u)\right. \\
& \left.-\mathscr{I}_{1, \text { upper }}^{-}(0, Y, u)\right]_{u=a}^{b}=\frac{1}{L} \sum_{n=0}^{\infty} \frac{\left(-Y^{2} / 2\right)^{n}}{n !} \\
& \cdot e^{-\operatorname{sgn}(a) u} u^{1-n}\left[K_{n}(u)\left(\frac{\operatorname{sgn}(a)(X+L)}{1-2 n}-\frac{u}{3-2 n}\right)\right. \\
& \left.+K_{n-1}(u)\left(\frac{1+\operatorname{sgn}(a) u}{3-2 n}-\frac{X+L}{1-2 n}\right)\right]_{u=|a|}^{|b|}
\end{aligned}
$$


Similarly, from (64), define

$$
\begin{gathered}
\left.\mathbf{I}_{\text {lower }}^{1}(Y, u)\right|_{u=a} ^{b}=\frac{1}{L}\left[(X+L) \mathscr{I}_{0, \text { lower }}^{-}(0, Y, u)\right. \\
\left.-\mathscr{I}_{1, \text { lower }}^{-}(0, Y, u)\right]_{u=a}^{b}=\frac{1}{L} \sum_{n=0}^{\infty} \frac{(-2 Y)^{-n}}{n !} K_{n}(Y) \\
\cdot[(X+L) \gamma(2 n+1, u)-\gamma(2 n+2, u)]_{u=a}^{b} .
\end{gathered}
$$

By using the recursion formula [3, Eqn 45:5:1]

$$
\gamma(\nu+1, x)=\nu \gamma(\nu, x)-x^{\nu} e^{-x},
$$

we reduce $(76)$ to

$$
\begin{aligned}
& \left.\mathbf{I}_{\text {lower }}^{1}(Y, u)\right|_{u=a} ^{b}=\frac{1}{L} \sum_{n=0}^{\infty} \frac{(-2 Y)^{-n}}{n !} K_{n}(Y) \\
& \quad \cdot\left[(X+L-2 n-1) \gamma(2 n+1, u)+u^{2 n+1} e^{-u}\right]_{u=a}^{b} .
\end{aligned}
$$

For the parabolic case, we can follow similar steps as in the linear case, arriving at

$$
\begin{gathered}
\left.\mathbf{I}_{\text {upper }}^{2}(X, Y, u)\right|_{u=a} ^{b}=\frac{3}{4 L^{2}} \sum_{n=0}^{\infty} \frac{\left(-Y^{2} / 2\right)^{n}}{n !} e^{-\operatorname{sgn}(a) u} u^{1-n} \\
\cdot\left\{K _ { n } ( u ) \left[\operatorname{sgn}(a) \frac{(X+L)^{2}}{1-2 n}\right.\right.
\end{gathered}
$$

$$
\begin{aligned}
& \left.-\frac{2 u}{3-2 n}\left(\frac{1}{5-2 n}+X+L\right)+\frac{\operatorname{sgn}(a) u^{2}}{5-2 n}\right] \\
& +K_{n-1}(u)\left[\frac{-(X+L)^{2}}{1-2 n}\right. \\
& +\frac{2(1+\operatorname{sgn}(a) u)}{3-2 n}\left(X+L-\frac{2(1-n)}{5-2 n}\right) \\
& \left.\left.-\frac{u^{2}}{5-2 n}\right]\right\}_{u=|a|}^{|b|}, \quad a b>0,
\end{aligned}
$$

$$
\begin{aligned}
& \left.\mathbf{I}_{\text {lower }}^{2}(Y, u)\right|_{u=a} ^{b}=\frac{3}{4 L^{2}} \sum_{n=0}^{\infty} \frac{K_{n}(Y)}{(-2 Y)^{n} n !}\{\gamma(2 n+1, u) \\
& \cdot\left[(X+L)^{2}+2(2 n+1)(n+1-X-L)\right] \\
& \left.+[2(X+L-n-1)-u] u^{2 n+1} e^{-u}\right\}_{u=a}^{b} .
\end{aligned}
$$

Finally, taking into account (66) and using the above results for $\mathbf{I}_{\text {upper }}^{m}$ and $\mathbf{I}_{\text {lower }}^{m}$, the dimensionless temperature field $\mathscr{T}_{m}(X, Y)$ for $m=0,1,2$, is calculated as follows:

$$
\mathscr{T}_{m}(X, Y)=\left.\mathbf{I}_{m}(X, Y, u)\right|_{u=X-L} ^{X+L}
$$

where

$$
\left.\mathbf{I}_{m}\right|_{a} ^{b}= \begin{cases}\left.\mathbf{I}_{\text {upper }}^{m}\right|_{a} ^{b}, & \left\{\begin{array}{l}
a>Y \text { or } \\
b<-Y
\end{array}\right. \\
\left.\mathbf{I}_{\text {upper }}^{m}\right|_{\min (a,-Y)} ^{-Y}+\left.\mathbf{I}_{\text {lower }}^{m}\right|_{\max (a,-Y)} ^{\min (b, Y)}+\left.\mathbf{I}_{\text {upper }}^{m}\right|_{Y} ^{\max (Y, b)}, & \left\{\begin{array}{l}
a<Y, \\
b>-Y .
\end{array}\right.\end{cases}
$$

Therefore,

$$
\begin{aligned}
& \mathscr{T}_{m}(X, Y) \\
& = \begin{cases}\left.\mathbf{I}_{\text {upper }}^{m}\right|_{X-L} ^{X+L}, & |X|>|Y+L| \\
\left\{\begin{array}{l}
\left.\mathbf{I}_{\text {upper }}^{m}\right|_{\min (X-L,-Y)} ^{-Y} \\
+\left.\mathbf{I}_{\text {upper }}^{m}\right|_{Y} ^{\max (Y, X+L)},
\end{array}\right. & |X|<|Y+L|,\end{cases}
\end{aligned}
$$

For the case of a triangular heat flux profile, according to (32) and (36) and taking into account (72) and (81), we have

$$
\begin{aligned}
& \mathscr{T}_{\lambda, L}(X, Y)=\frac{2}{L(1+\lambda)}\left\{(X+L) \mathscr{I}_{0}^{-}(0, Y, u)\right. \\
& \left.-\mathscr{I}_{1}^{-}(0, Y, u)\right\}_{u=X-\lambda L}^{X+L}=\left.\frac{2}{1+\lambda} \mathbf{I}_{1}(X, Y, u)\right|_{u=X-\lambda L} ^{X+L} .
\end{aligned}
$$

It is worth noting that $\left.\mathbf{I}_{1}\right|_{a} ^{b}$ is calculated properly with the formulas given for $\mathbf{I}_{\text {upper }}^{1}$ and $\mathbf{I}_{\text {lower }}^{1}$ in (75) and (78) when $a<b$. Therefore, care has to be taken when we compute the dimensionless temperature for the triangular case as $\mathscr{T}_{\Delta}(X, Y)=\mathscr{T}_{\lambda, L}(X, Y)-\mathscr{T}_{-\lambda,-L}(X, Y)$, as stated in (31).

Notice also that, in order to calculate the dimensionless temperature $\mathscr{T}(X, Y)$, we need to compute the series given in (70), (75), and (79) for $\mathbf{I}_{\text {upper }}^{m}$ and (71), (78), and (80) for $\mathbf{I}_{\text {lower. }}^{m}$. These series are all alternating series, which converges slowly when $|u| \approx Y$. However, we can accelerate the convergence of these alternating series by using Cohen-Villegas-Zagier algorithm [20]. This algorithm approximates an alternating series $S=\sum_{k=0}^{\infty}(-1)^{k} a_{k}$ as a weighted sum of the first $n$ values of $a_{k}$ by using a "Padé type approximation," as long as $a_{k}$ is a reasonable well-behaved sequence. We will see in Section 6 that we need a small number of terms $n$ in order to get a good accuracy.

4.5. Temperature on the Surface. As mentioned before in the Introduction, $\mathscr{T}(X, 0)$ can be calculated in closed form for the 
heat flux profiles considered in this paper. All these results can be obtained from the results of Section 4 taking $Y=0$. As a consistency test, we are going to derive here the expression given in (12) for a constant heat flux profile. For this purpose, note that when $y=0$, the series given for $\mathscr{J}_{0 \text {,lower }}^{ \pm}$, that is, (47), vanishes; thus, according to (35), we have

$$
\left.\mathscr{F}_{0}^{ \pm}(\nu, 0, u)\right|_{u=a} ^{b}=\left.\mathscr{I}_{0, \text { upper }}^{ \pm}(\nu, 0, u)\right|_{u=a} ^{b}
$$

Now, taking $y=0$ in (69) and applying the property $K_{\nu}(z)=K_{-\nu}(z)[19$, Eqn. 5.7.10], (85) is reduced to

$$
\begin{aligned}
&\left.\mathscr{I}_{0}^{ \pm}(0,0, u)\right|_{u=a} ^{b}=\left.u e^{ \pm u}\left[K_{0}(u) \pm K_{1}(u)\right]\right|_{u=a} ^{b}, \\
& a, b>0 .
\end{aligned}
$$

According to the limiting forms [17, Eqn. 10.3.2-3],

$$
\begin{aligned}
& K_{\nu}(z) \approx \frac{1}{2} \Gamma(\nu)\left(\frac{z}{2}\right)^{-v}, \quad z \longrightarrow 0, \operatorname{Re} v>0, \\
& K_{0}(z) \approx-\log z, \quad z \longrightarrow 0,
\end{aligned}
$$

we have

$$
\lim _{u \rightarrow 0^{+}} u e^{ \pm u}\left[K_{0}(u) \pm K_{1}(u)\right]= \pm 1
$$

so that

$$
\begin{aligned}
\left.\mathscr{I}_{0}^{ \pm}(0,0, u)\right|_{u=0} ^{z}=z e^{ \pm z}\left[K_{0}(z) \pm K_{1}(z)\right] \mp 1, & z>0, \\
\left.\lim _{z \rightarrow 0^{+}} \mathscr{I}_{0}^{ \pm}(0,0, u)\right|_{u=0} ^{z}=0 . &
\end{aligned}
$$

Now, according to (85), apply (51), exchange the integration limits, and take into account (89), to arrive at

$$
\begin{aligned}
\left.\mathscr{I}_{0}^{ \pm}(0,0, u)\right|_{u=0} ^{-z} & =-\left.\mathscr{I}_{0}^{\mp}(0,0, u)\right|_{u=0} ^{z} \\
& =-z e^{\mp z}\left[K_{0}(z) \mp K_{1}(z)\right] \mp 1,
\end{aligned}
$$

thus, performing the change $z \rightarrow-z$,

$$
\begin{aligned}
\left.\mathscr{F}_{0}^{ \pm}(0,0, u)\right|_{u=0} ^{z}=z e^{ \pm z}\left[K_{0}(-z) \mp K_{1}(-z)\right] \mp & 1, \\
& z<0 .
\end{aligned}
$$

Collecting the results (89), (90), and (92), we arrive at

$$
\begin{aligned}
& \left.\mathscr{F}_{0}^{ \pm}(0,0, u)\right|_{u=0} ^{z} \\
& \quad= \begin{cases}z e^{ \pm z}\left[K_{0}(|z|) \pm \operatorname{sgn}(z) K_{1}(|z|)\right] \mp 1, & z \neq 0 \\
0, & z=0 .\end{cases}
\end{aligned}
$$

Finally, according to (67) and applying again (51), the dimensionless temperature on the surface is

$$
\begin{aligned}
\mathscr{T}_{0}(X, 0) & =\left.\mathscr{F}_{0}^{-}(0,0, u)\right|_{u=X-L} ^{X+L} \\
& =\left.\mathscr{F}_{0}^{+}(0,0, u)\right|_{u=-X-L} ^{-X+L},
\end{aligned}
$$

which is equivalent to (12), taking into account (93). Therefore, for the computation of the temperature field with the series expansions given above, we will use directly the closed form formulas given in (12) and (14)-(16).

\section{Maximum Temperature Beneath the Surface}

As aforementioned in the Introduction, we present in this section a numerical method to compute the maximum temperature as a function of the Peclet number $L$ and the dimensionless depth $Y$ below the surface, that is, $\mathscr{T}_{0 \text {,max }}(Y, L)$. For this purpose, note that the integrand of (18) is positive, since $\forall z>0, K_{0}(z)>0[19$, Note 38$]$. Therefore,

$$
\mathscr{T}_{0}(X, Y)=\int_{X-L}^{X+L} e^{-u} K_{0}\left(\sqrt{u^{2}+Y^{2}}\right) d u>0,
$$

$X \in \mathbb{R}$.

Also, directly from (95), we have

$$
\lim _{X \rightarrow \pm \infty} \mathscr{T}_{0}(X, Y)=0 .
$$

The behavior of $\mathscr{T}_{0}(X, Y)$ given in (95) and (96) is shown graphically in Figure 3. Moreover, for each $Y, \mathscr{T}_{0}(X, Y)$ exhibits and unique extreme value that matches the maximum value. Therefore, we can search for the maximum temperature at a given depth $Y$ looking for the extreme value of $\mathscr{T}_{0}(X, Y)$ as a function of $X$, solving for $X_{\max }$ the equation

$$
\frac{\partial \mathscr{T}_{0}\left(X_{\max }, Y\right)}{\partial X}=0 .
$$

By using Leibniz's theorem for differentiation of integrals [17, Eqn. 1.5.22]

$$
\begin{aligned}
\frac{d}{d x} \int_{\alpha(x)}^{\beta(x)} f(x, y) d y= & f(x, \beta(x)) \beta^{\prime}(x) \\
& -f(x, \alpha(x)) \alpha^{\prime}(x) \\
& +\int_{\alpha(x)}^{\beta(x)} \frac{\partial f}{\partial x} d y .
\end{aligned}
$$

Equation (97) reads as

$$
\begin{aligned}
e^{-L} K_{0}\left(\sqrt{\left(X_{\max }+L\right)^{2}+Y^{2}}\right) \\
=e^{L} K_{0}\left(\sqrt{\left(X_{\max }-L\right)^{2}+Y^{2}}\right),
\end{aligned}
$$

which has to be solved numerically. Taking as initial iteration point $X_{\max }^{(0)}=0$, Newton's method for root searching converges quite rapidly. Notice that $X_{\max }=X_{\max }(Y, L)$; thus the maximum temperature $\mathscr{T}_{0 \text {,max }}$ is calculated as a function of the dimensionless depth $Y$ and the Peclet number $L$,

$$
\mathscr{T}_{0, \text { max }}(Y, L)=\mathscr{T}_{0}\left(X_{\text {max }}(Y, L), Y\right) .
$$

In Section 6, we compare this numerical approach with Takazawa's approximation (19). 
TABLE 2: Simulation parameters in SI units.

\begin{tabular}{|c|c|c|c|c|}
\hline & & Data 1 & Data 2 & Data 3 \\
\hline \multirow{3}{*}{ Workpiece } & Material & Sapphire & Steel & Titanium \\
\hline & $k_{0}$ & 46 & 61 & 13 \\
\hline & $k$ & $1.51 \times 10^{-5}$ & $1.78 \times 10^{-5}$ & $4.23 \times 10^{-6}$ \\
\hline \multirow{4}{*}{ Grinding regime } & $2 \ell$ & $1.4 \times 10^{-3}$ & $2.5 \times 10^{-3}$ & $2.66 \times 10^{-3}$ \\
\hline & $q$ & $1.4 \times 10^{7}$ & $3.5 \times 10^{7}$ & $5.89 \times 10^{7}$ \\
\hline & $v_{d}$ & $3.3 \times 10^{-2}$ & 0.15 & 0.53 \\
\hline & $T_{\infty}$ & 300 & 300 & 300 \\
\hline \multirow{2}{*}{ Dimensionless parameters } & $L$ & 0.76 & 5.27 & 83.4 \\
\hline & $Y_{0.1}(L)$ & 1.68 & 4.42 & 17.6 \\
\hline
\end{tabular}

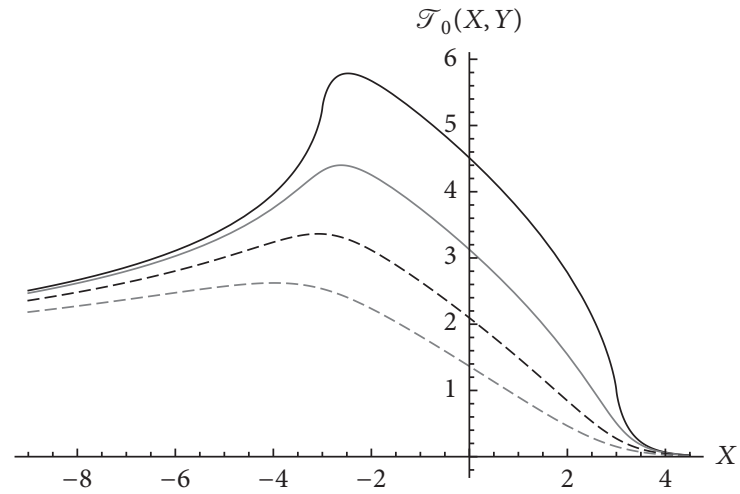

Y

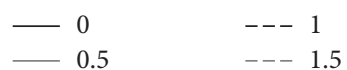

Figure 3: $\mathscr{T}_{0}(X, Y)$ for different dimensionless depths $Y$ and Peclet number $L=3$.

\section{Numerical Results}

In this section, we compare the performance of the numerical evaluation of the temperature field by using the integral form (7) with the series expansion derived in Section 4, namely, (83) and (84). Also, we analyze the accuracy of Takazawa's approximation for the maximum temperature (19), by using the numerical method derived in Section 5. For both purposes, we need to define first the characteristic dimensionless depth of the temperature field in dry grinding.

6.1. Characteristic Dimensionless Depth. According to the one-dimensional approximation, the depth of thermal penetration for dry grinding is given by

$$
\delta_{p}=2 g^{-1}(p) \sqrt{k \frac{2 \ell}{v_{d}}},
$$

where the function $g^{-1}(p)$ is the inverse function of the following function:

$$
g(x)=e^{-x^{2}}-\sqrt{\pi} x \operatorname{erfc}(x)
$$

and $p$ is the percentage at which the temperature falls at depth $\delta_{p}$ with respect to the surface temperature (i.e., at $\delta_{0.1}$ below the surface, the temperature is $10 \%$ of the surface temperature). Equation (101) provides a very good approximation to the two-dimensional depth of thermal penetration for moderate values of $p$, that is, $0.3 \leqslant p \leq 1$, and the heat flux profiles presented in Figure 2. This depth of thermal penetration $\delta_{p}$ is useful to set the characteristic domain of the temperature field, and thereby to compare the performance of the integral form with the series expansion of the temperature field.

From (101), we define the characteristic dimensionless depth as

$$
Y_{p}(L)=\frac{\delta_{p}}{s}=2 g^{-1}(p) \sqrt{L}
$$

where we remember that $s$ is the characteristic length given in (6). This characteristic dimensionless depth $Y_{p}(L)$ is very useful to analyze the accuracy of Takazawa's approximation for the maximum temperature (19).

6.2. Temperature Field Computation. Table 2 shows three sets of parameters (in SI units) for the numerical simulations. Data set 1 considers a carbon steel workpiece AISI 1020 [21] and data set 2 considers aluminum oxide, $\mathrm{Al}_{2} \mathrm{O}_{3}$ (sapphire), as workpiece material [22]. Also, data set 3 considers a titanium alloy VT20 workpiece, whose thermal properties are given in [23]. The grinding regime for this simulation can be found in [24]. These simulation parameters have been chosen in order to cover a wide range of Peclet numbers $L$. The characteristic dimensionless depth $Y_{0.1}(L)$ of the temperature field is given as well for the three data sets.

Figure 4 shows the temperature field for data set 1, where we have considered as plot area

$$
D=[-1.5 \ell, 1.5 \ell] \times\left[0, \delta_{0.1}\right]
$$

In order to compare the performance of the numerical integration of (7) and the numerical evaluation of the series expansions given in Section 4 for the calculation of the temperature field, we have set the time ratio $\tau$ as the ratio between the computation time of the numerical integration $t_{\text {integration }}$ and the series expansion $t_{\text {series, }}$,

$$
\tau=\frac{t_{\text {integration }}}{t_{\text {series }}} .
$$


TABLE 3: Relative distances $\Delta_{D}$ and time ratios $\tau$ between the integration method and the series method, where $n$ denotes the number of terms taken in the series approximation.

\begin{tabular}{|c|c|c|c|c|}
\hline Heat flux & & Data 1 & Data 2 & Data 3 \\
\hline \multirow{3}{*}{ Constant } & $\Delta_{D}$ & $2.34 \times 10^{-8}$ & $4.26 \times 10^{-7}$ & $2.86 \times 10^{-5}$ \\
\hline & $\tau$ & 7.43 & 7.47 & 7.45 \\
\hline & $n$ & 8 & 8 & 10 \\
\hline \multirow{3}{*}{ Linear } & $\Delta_{D}$ & $3.41 \times 10^{-8}$ & $7.57 \times 10^{-7}$ & $2.56 \times 10^{-5}$ \\
\hline & $\tau$ & 6.40 & 6.15 & 5.45 \\
\hline & $n$ & 8 & 8 & 10 \\
\hline \multirow{3}{*}{ Triangular } & $\Delta_{D}$ & $2.34 \times 10^{-8}$ & $7.01 \times 10^{-7}$ & $2.56 \times 10^{-5}$ \\
\hline & $\tau$ & 17.16 & 17.10 & 11.73 \\
\hline & $n$ & 8 & 8 & 10 \\
\hline \multirow{3}{*}{ Parabolic } & $\Delta_{D}$ & $4.26 \times 10^{-8}$ & $1.07 \times 10^{-6}$ & $2.33 \times 10^{-5}$ \\
\hline & $\tau$ & 5.06 & 5.50 & 1.63 \\
\hline & $n$ & 8 & 8 & 10 \\
\hline
\end{tabular}

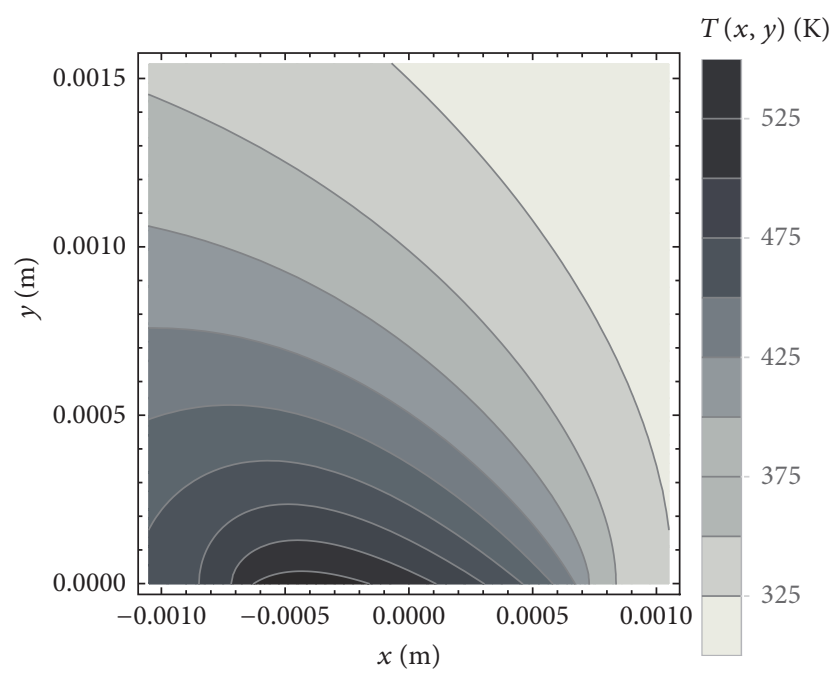

FIGURE 4: Temperature field for data set 1 and a constant heat flux profile.

$t_{\text {integration }} \approx 10-25 \mathrm{~s}$ for plotting the temperature field (i.e., Figure 4) running an 8 kernel i7 CPU computer with MATHEMATICA $^{\mathrm{TM}}$ on Linux platform. However, this time could be increased by a factor of 2-5 times in the numerical control of the grinding machines; thus a time ratio $\tau \geq 2$ would be worthwhile.

Also, to compare both temperature fields, we have considered the following distance between two nonnegative scalar fields $f$ and $g$, integrable in a certain connected open set $D \subseteq \mathbb{R}^{n}[25]$

$$
\begin{array}{ll}
\Delta_{D}(f, g) \\
= \begin{cases}\frac{\int_{D}|f(\vec{x})-g(\vec{x})| d V}{\int_{D}[f(\vec{x})+g(\vec{x})] d V}, & \text { for } g \neq 0 \\
0, & f=g=0 .\end{cases}
\end{array}
$$

This distance $\Delta_{D}$ is applicable to the temperature field since, according to (95); the latter is a positive quantity. In [25], it is proved that $0 \leq \Delta_{D}(f, g) \leq 1$, wherein $\Delta_{D}(f, g)=0$ means that $f$ and $g$ are overlapped within the integration domain $D$, and $\Delta_{D}(f, g)=1$ means that both functions are relatively infinitely far one from each other. Table 3 shows the distances $\Delta_{D}$ between the temperature fields computed by the numerical integration of (7) and the numerical evaluation of the corresponding series (83) and (84), taking as integration domain (104).

To evaluate numerically the alternating series $S=$ $\sum_{k=0}^{\infty}(-1)^{k} a_{k}$ given in (70), (75), and (79) for $\mathbf{I}_{\text {upper }}^{m}$ and (71), (78), and (80) for $\mathbf{I}_{\text {lower }}^{m}$, we have taken $n=8$ terms in the Cohen-Villegas-Zagier algorithm [20] for data sets 1 and 2 and $n=10$ for data set 3 . In particular, we have used the $2_{A}$ algorithm, which consists first of computing and storing in memory the polynomial

$$
A_{n}\left(\sin ^{2} t\right)=\frac{1}{2^{n} n !} D^{n}\left(\sin ^{n} 2 t\right)
$$

and then we apply the following procedure.

Algorithm $1\left(2_{A}\right)$. Let $A_{n}(x)=\sum_{k=0}^{n} b_{k} x^{k}$. Set $d=-A_{n}(-1)$; $c=-d ; s=0$. For $k=0$ up to $k=n-1$, repeat: $c=b_{k}-c$; $s=s+c a_{k}$. Output: $s / d$.

It is worth noting that there is a typo in the algorithm published in [20], where $d$ is given as $A_{n}(-1)$.

Table 3 shows the time ratios for the three data sets. We can see that the computation of the corresponding series expansions of Section 4 is considerably faster than the numerical integration. This is especially true for a triangular heat flux profile, for which we have set $\lambda=0.5$ (similar results are found for different values of $\lambda$ ). Also, both methods provide very similar temperature fields, since $\Delta_{D} \ll 1$. This is especially true for low Peclet numbers.

6.3. Takazawa's Approximation Analysis. We can compare the exact value given in (100) with the approximation given by Takazawa in (19). Figure 5 shows this comparison for 


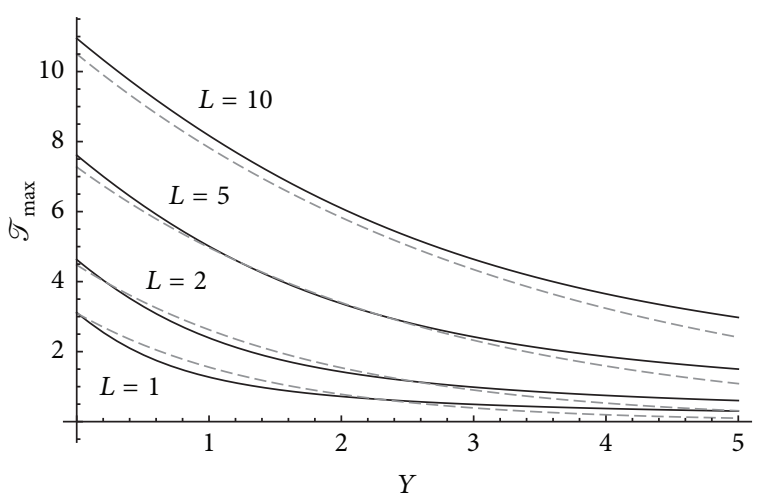

- Numeric

--- Takazawa

FIgURE 5: $\mathscr{T}_{0, \max }(Y)$ for intermediate Peclet numbers.

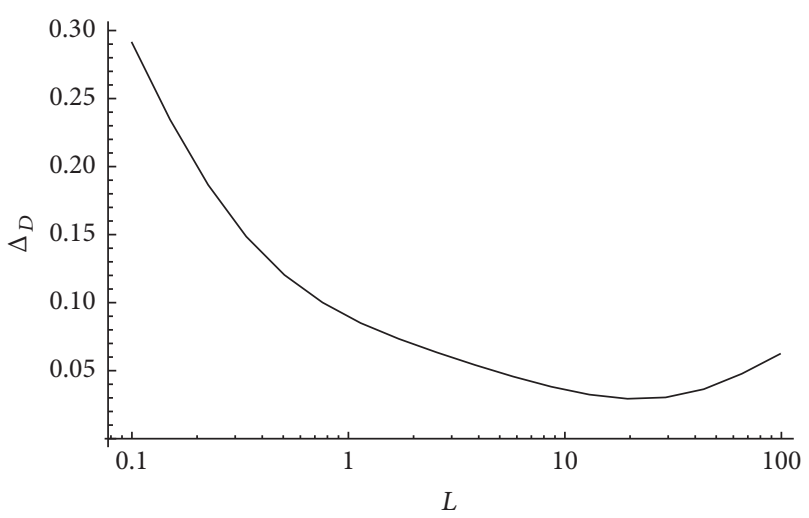

Figure 6: Accuracy of Takazawa's approximation.

different values of the Peclet number. We can appreciate that, regardless of the value of the Peclet number, Takazawa's approximation deviates from the exact value for large $Y$.

In order to measure the accuracy of Takazawa's approximation (19), we can compute the relative distance $\Delta_{D}$ of (19) with respect to the numerical value (100) as a function of the Peclet number $L$, taking as integration domain $D=$ $\left[0, Y_{0.1}(L)\right]$. Figure 6 presents this computation, showing that the accuracy of Takazawa's approximation is in general poor, especially for low Peclet numbers. Therefore, despite the fact Takazawa's formula being extremely easy to compute, probably it is better to use the numerical method given in (100), since the numerical evaluation of $X_{\max }(Y, L)$ is very rapid, and the computation of $\mathscr{T}(X, Y)$ is quite fast by using the series expansion method described in Section 4. For instance, Figure 5 is plotted in $\approx 0.87 \mathrm{~s}$ by using MATHEMATICA and an Intel i7 CPU.

\section{Conclusions}

In the framework of Jaeger's model for heat transfer in dry surface grinding, we consider the integral formula for the dimensionless temperature field of the workpiece, assuming the most common heat flux profiles reported in the literature.
On the one hand, series expansions of the temperature field in dry grinding for the heat flux profiles considered have been calculated. By using the Cohen-Villegas-Zagier algorithm for computing alternating series, the numerical evaluation of these series expansions is considerably faster than the numerical integration of the integral form given in Jaeger's model for a wide range of Peclet numbers. The latter is especially true in the case of a triangular heat flux profile, for which the series method is $\approx 17$ times faster than the numerical integration. Also, these series expansions provide an evaluation of the temperature field as accurate as the integral form within the domain where the temperature rise is more significant. The latter is especially true for low Peclet numbers.

On the other hand, considering a constant heat flux profile, a numerical procedure for the computation of the maximum temperature as a function of the Peclet number and the depth below the surface has been proposed. This numerical procedure has been used to evaluate the accuracy of Takazawa's approximation.

\section{Conflicts of Interest}

The author declares that they have no conflicts of interest.

\section{Acknowledgments}

The author wish to thank the financial support received from Universidad Católica de Valencia under Grants PRUCV/2015/612 and 2017-160-001.

\section{References}

[1] H. S. Carslaw and J. C. Jaeger, Conduction of heat in solids, Clarendon Press, Oxford, UK, 1988.

[2] S. Malkin and C. Guo, Grinding Technology: Theory and Application of Machining with Abrasives, Industrial Press Inc, New York, NY, USA, 2008.

[3] K. Oldham, J. Myland, and J. Spanier, An Atlas of functions: with equator, the Atlas function calculator, Springer, New York, NY, USA, 2nd edition, 2008.

[4] A. S. Lavine, S. Malkin, and T. C. Jen, "Thermal aspects of grinding with CBN wheels," CIRP Annals - Manufacturing Technology, vol. 38, no. 1, pp. 557-560, 1989.

[5] C. Guo and S. Malkin, "Analysis of energy partition in grinding," Journal of Engineering for Industry, vol. 117, no. 1, pp. 55-61, 1995.

[6] W. J. Sauer, “Thermal aspects of surface grinding," in Proceedings of International Grinding Conference, pp. 391-411, 1972.

[7] C. Guo, Y. Wu, V. Varghese, and S. Malkin, “Temperatures and energy partition for grinding with vitrified CBN wheels," CIRP Annals - Manufacturing Technology, vol. 48, no. 1, pp. 247-250, 1999.

[8] D. Anderson, A. Warkentin, and R. Bauer, "Experimental validation of numerical thermal models for dry grinding," Journal of Materials Processing Technology, vol. 204, no. 1-3, pp. 269-278, 2008.

[9] M. Mahdi and L. Zhang, "Applied mechanics in grinding-VI. Residual stresses and surface hardening by coupled thermoplasticity and phase transformation," The International Journal 
of Machine Tools and Manufacture, vol. 38, no. 10-11, pp. 12891304, 1998.

[10] I. Zarudi and L. C. Zhang, "A revisit to some wheel-workpiece interaction problems in surface grinding," The International Journal of Machine Tools and Manufacture, vol. 42, no. 8, pp. 905-913, 2002.

[11] W. B. Rowe, S. C. E. Black, B. Mills, H. S. Qi, and M. N. Morgan, "Experimental investigation of heat transfer in grinding," CIRP Annals - Manufacturing Technology, vol. 44, no. 1, pp. 329-332, 1995.

[12] A. Brosse, P. Naisson, H. Hamdi, and J. M. Bergheau, “Temperature measurement and heat flux characterization in grinding using thermography," Journal of Materials Processing Technology, vol. 201, no. 1-3, pp. 590-595, 2008.

[13] J. L. González-Santander, "Calculation of an integral arising in dry flat grinding for a general heat flux profile. Application to maximum temperature evaluation," Journal of Engineering Mathematics, vol. 88, pp. 137-160, 2014.

[14] J. C. Jaeger, "Moving sources of heat and the temperature at sliding contacts," Journal and Proceedings of the Royal Society of New South Wales, vol. 76, pp. 204-224, 1942.

[15] K. Takazawa, "Effects of grinding variables on surface structure of hardened steel," B Jpn Soc Prec Eng, vol. 2, no. 1, pp. 14-21, 1966.

[16] Y. A. Brychkov, Handbook of special functions: derivatives, integrals, series and other formulas, CRC Press, New York, NY, USA, 2008.

[17] F. W. J. Olver, D. W. Lozier, R. F. Boisvert, and C. W. Clark, Eds., NIST Handbook of Mathematical Functions, Cambridge University Press, Cambridge, UK, 2010.

[18] M. R. Spiegel, Handbook of Mathematical Formulas, SchaumMcGraw Hill, New York, NY, USA, 1968.

[19] N. N. Lebedev, Special Functions and Their Applications, Prentice-Hall, 1965.

[20] H. Cohen, F. Rodriguez Villegas, and D. Zagier, "Convergence acceleration of alternating series," Experimental Mathematics, vol. 9, no. 1, pp. 3-12, 2000.

[21] C. Guo and S. Malkin, "Inverse heat transfer analysis of grinding, part 2: applications," J Eng Ind-T ASME, vol. 118, no. 1, pp. 143-149, 1996.

[22] A. S. Lavine, "A simple model for convective cooling during the grinding process," Journal of Engineering for Industry, vol. 110, no. 1, pp. 1-6, 1988.

[23] V. I. Murav'ev, A. V. Yakimov, and A. V. Chernyshev, "Effect of deformation, welding, and electrocontact heating on the properties of titanium alloy VT20 in pressed and welded structures," Metal Science and Heat Treatment, vol. 45, no. 11-12, pp. 419-422, 2003.

[24] D. L. Skuratov, Y. L. Ratis, I. A. Selezneva, J. Pérez, P. F. de Córdoba, and J. F. Urchueguía, "Mathematical modelling and analytical solution for workpiece temperature in grinding," Applied Mathematical Modelling, vol. 31, no. 6, pp. 1039-1047, 2007.

[25] J. L. González-Santander and G. M. González, "Relative distance between two scalar fields. Application to mathematical modelling approximation," Mathematical Methods in the Applied Sciences, vol. 37, no. 18, pp. 2906-2922, 2014. 


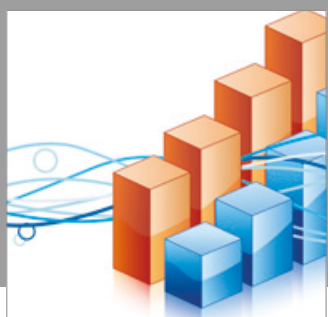

Advances in

Operations Research

vatersals

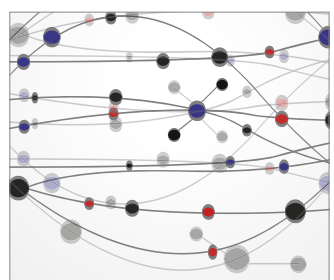

\section{The Scientific} World Journal
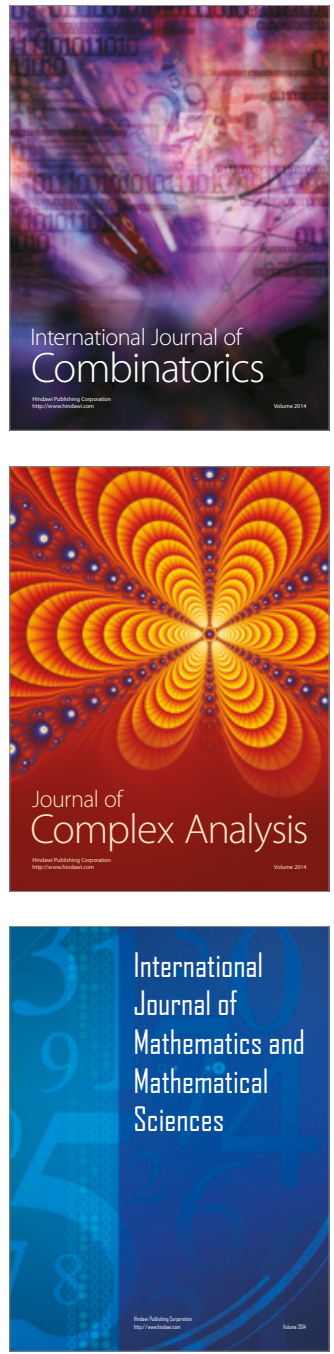
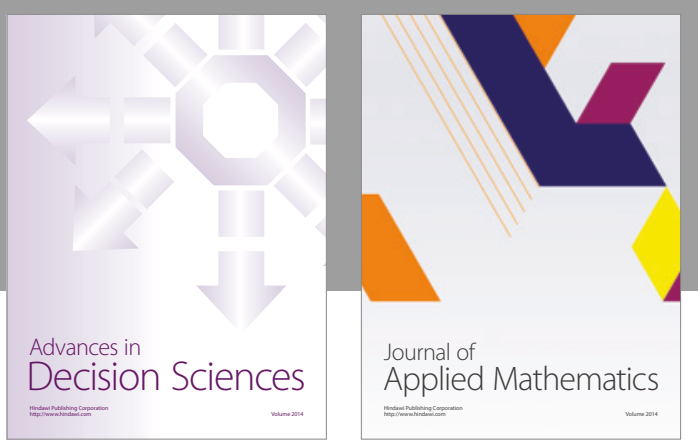

Algebra

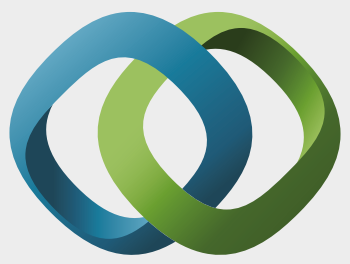

\section{Hindawi}

Submit your manuscripts at

https://www.hindawi.com
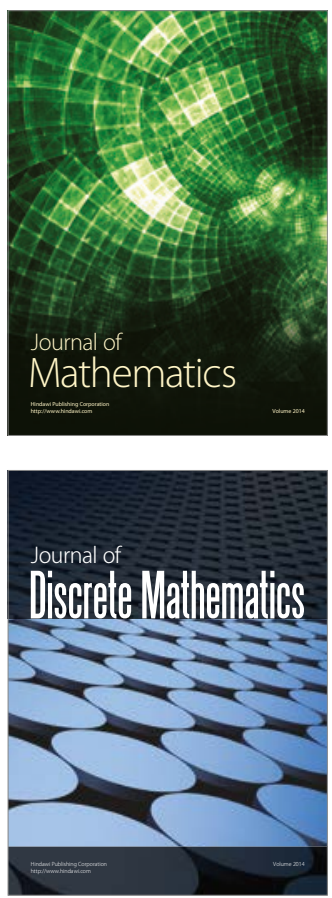

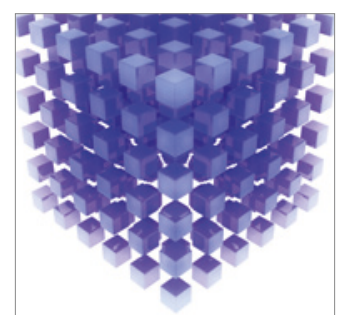

Mathematical Problems in Engineering
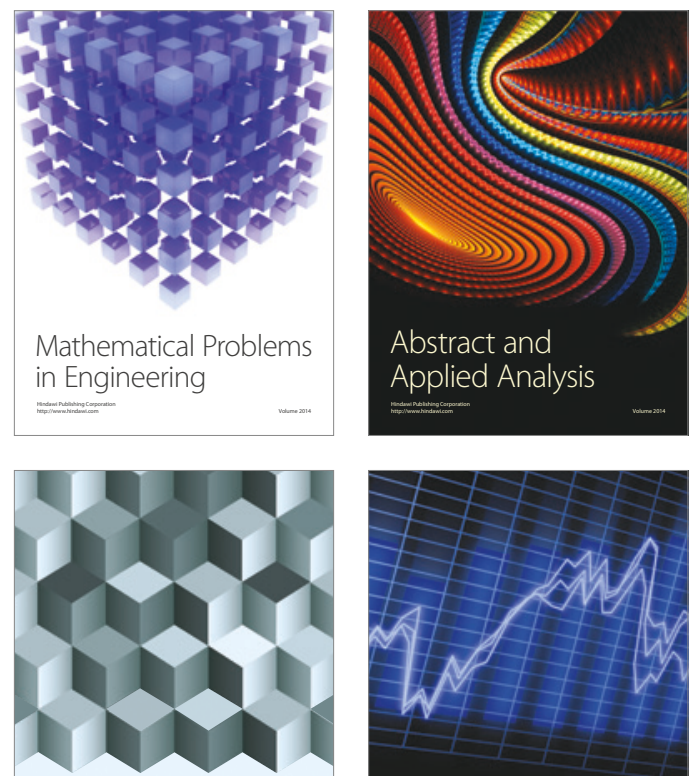

Journal of

Function Spaces

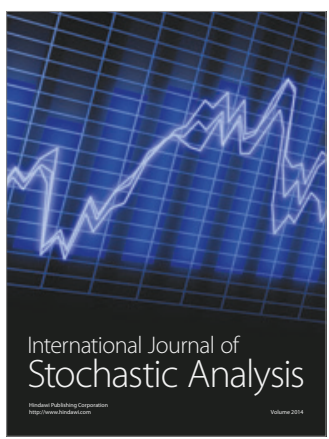

Probability and Statistics
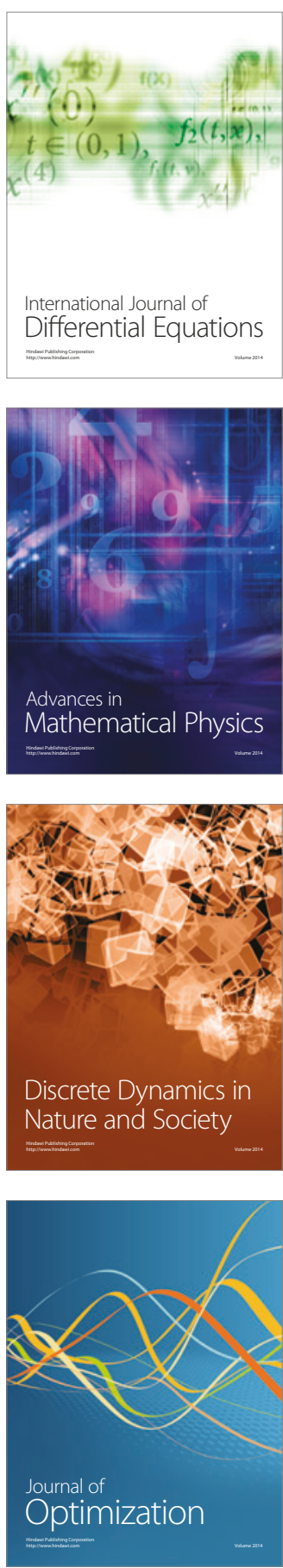